\title{
Percutaneous Radiofrequency Lesioning of Sensory Branches of the Obturator and Femoral Nerves for the Treatment Of Non-OPERABle Hip PaIN
}

Atif Malik, MD, Thomas Simopolous, MD, Mohamed Elkersh, MD, Musa Aner, MD, and Zahid H. Bajwa, MD

\begin{abstract}
Background: Chronic hip pain is often a debilitating problem and many patients are not good surgical candidates. Furthermore, hip replacement surgery has significant associated risks. We offer a conservative approach to hip replacement using radiofrequency lesioning.

Methods: This is a case series of 4 patients who had anterior and or medial hip pain who underwent percutaneous radiofrequency lesioning of the sensory branches of the obturator and femoral nerves. All of the patients had significant co-morbidities and the risks out weighed the benefits of sur-
\end{abstract}

gery. All the patients had diagnostic blocks with local anesthetic to the sensory articular branches of the obturator and femoral nerves and showed temporary benefit. Continuous radiofrequency lesioning was performed from $60^{\circ} \mathrm{C}$ to $80^{\circ} \mathrm{C}$ for 90 seconds. The outcome measures were improvement in visual analogue scale (VAS), improvement in function and the decreased use of pain medications.

Results: All four patients had reduction in pain, while 3 of 4 patients had improved functioning. Two of the four patients had decreased use of their pain medication. One of the four patients reported numbness at the hip and there were no other side effects.

Conclusion: Percutaneous radiofrequency lesioning of the sensory branches of the obturator and femoral nerves appears to be a safe alternative to hip replacement, especially in those patients where surgery is not an option. Further studies are needed to confirm our results.

Keywords: Hip joint pain, radiofrequency of the sensory branches of the obturator and femoral nerves
Hip pain is a common condition that is often seen in the rehabilitation setting in patients with multiple co-morbidities. Often surgery is not an option and conventional drugs have either too many side effects or are ineffective. Percutaneous radiofrequency lesioning of the articular branches of the obturator and femoral nerves is a novel alternative treatment for hip pain that has been previously reported in literature (1).

The hip joint's nerve supply responsible for sensory input includes the gluteal, femoral, obturator and sciatic nerves. The innervation of the joint capsule can be divided into the anteromedial, anterior, posteromedial, posterior, and the posterolater$\mathrm{al}$ areas that are innervated by the articular branches of the obturator, femoral, sciatic and gluteal nerves, respectively $(2,3)$.

From Department of Anesthesia, Beth Israel Dea coness Medical Center, Harvard Medical School, Boston, Massachusetts. Address Correspondence: Atif Malik, MD, Arnold Pain Center, Department of Anesthesia, Beth Israel Deaconess Medical Center, Harvard Medical School, 330 Brookline Avenue, Boston, MA 02215.

E-mail: amalik@bidmc.harvard.edu

Disclaimer: None of the authors have any disclosures or grants. This material has not been pre sented or submitted previously.
The hip joint innervation has been well studied and has led some to examine the sensory nerve innervation of formalin-fixed hip joint capsules (3). It was clear that there is a separation between the anterior and posterior sensory innervation of the hip joint capsule. The anteromedial innervation is by the articular branches of the obturator nerve. Additionally, the anterior hip joint capsule is innervated by the sensory articular branches of the femoral nerve. The posterior portion has articular branches of the sciatic nerve innervate the posteromedial section of the hip joint capsule while the articular branches of the superior gluteal nerve innervate the posterolateral section of the hip joint capsule. From this anatomical study it is clear that a major portion of the hip joint receives its sensory supply form the obturator nerve and femoral nerves, but sciatic nerve and superior gluteal nerves also contribute (3).

\section{Methods}

In our case study we chose patients who demonstrated hip pain in the anterior and medial distribution. The patients were chosen after orthopedic consultation determined that these patients were not good candidates for joint replacement surgery based on their co-morbidities. We also ruled out patients who had low back pain or had posterior or buttock pain that could be attributed to causes other than hip joint pathology. Patients also had a diagnostic block of the obturator and femoral nerves using $1 \mathrm{ml}$ of $0.25 \%$ Marcaine, respectively at each location described below.

After a thorough explanation to the patients, consent was obtained for percutaneous radiofrequency of the sensory branches of the obturator and femoral nerves. After palpating for the femoral artery, the needle was inserted just medial to the femoral artery below the level of the inguinal ligament. Under fluoroscopy, the electrode was placed at the site below the inferior junction of the pubis and the ischium, which appears teardrop in shape in the anteroposterior radiograph (Fig. 1). The lesioning of the articular branches of femoral nerves were done via the electrode that was inserted through a $10 \mathrm{~mm}$ radiofrequency needle from a site below the anterior superior iliac spine to the anterolateral aspect of the extraarticular portion of the hip joint. The electrode tip was located below the anterior inferior iliac spine 


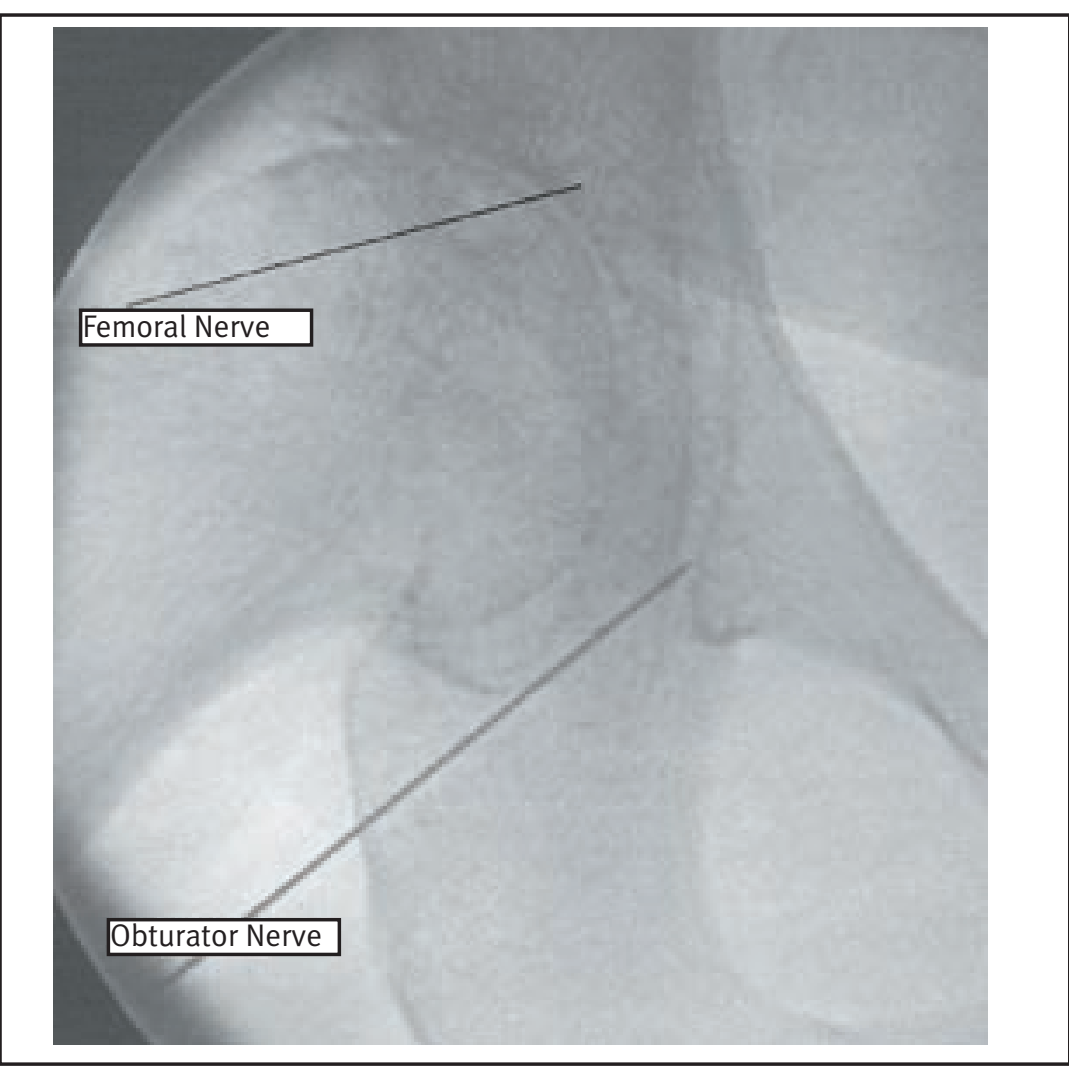

Fig 1. Fluoroscopic image of sites for RF lesioning of obturator and femoral nerves.

near the anterolateral margin of the hip joint. The radiofrequency thermocoagulation was performed at $75^{\circ} \mathrm{C}$ to $80^{\circ} \mathrm{C}$ for 90 seconds using an RFG-3B generator (Radionics, Burlington, MA).

The outcome measures that were determined were:

(1) The visual analog scale (VAS); a single question comprised of a $100-\mathrm{mm}$ line anchored with "no pain" and "the worst pain possible." To quantify the rating from 0 to 10 , the respondent places a mark across the line at the point that represents perceived pain intensity. A reduction of VAS score of more than $50 \%$ of the value before the procedures was defined as "effective."

(2) Improvement in function. This was collected from the patient through a series of questions where the patient indicated improvement in ambulation, improvement in ability to perform activities of daily living or change in their status of using assistive devices to help ambulate. Improvement in ambulation was graded ability to tolerate house-hold or community (shopping) level distance that was not possible prior to the procedure
(3) Decrease in use of pain medication was also used as an indicator of overall improvement.

All of the patients were followed up every four weeks (except one) and were asked a series of questions related to the radiofrequency procedure and outcome.

\section{Case Report \#1}

A 70-year-old female with chronic right hip pain on and off for 5 years was evaluated. She describes the pain as a constant dull achy discomfort in the groin at was $7 / 10$ on VAS. The pain was exacerbated by walking and limiting her ability to walk more than household distances. The pain is often sharp and starts in the hip and radiates to the thigh and groin area. She previously had hip joint injection with lidocaine and steroids that gave her transient relief for a few days. She was not a surgical candidate and her past medical history included idiopathic hypertrophic subaortic stenosis (IHSS), stroke, diabetes, headache, fibromyalgia, and sleep disorder. Her pain medication included tramadol $50 \mathrm{mg}$ 1-2 tablets up to four times a day. On physical exam she had pain with passive and active range of the right hip as well as tenderness over the greater trochanter. She had leg length discrepancy with her right leg being shorter by 2 centimeters. Otherwise the examination was normal. X-ray of the right hip showed severe degenerative changes and loss of joint space. She had four days of relief with diagnostic blocks to the right hip. She underwent radiofrequency lesioning of the obturator and femoral branches of the right hip. There were no complications or side effects from the procedure. At 4 weeks, she reported little to no pain. At 8 and 12 weeks her pain was $2 / 10$ and $4 / 10$, respectively. She reported that since the procedure she has been able to ambulate for longer distances outside her home and is no longer using a cane except at community distances. She now only uses tramadol on as needed basis, usually 1 or 2 tablets a day.

\section{Case Report \#2}

A 49-year-old man with s/p liver transplant rejection and severe liver failure from blood transfusion acquired hepatitis $\mathrm{C}$ after a gunshot wound. He suffered chronic avascular necrosis of the left hip. After a fall he then had a left subtrochanteric femur fracture and underwent s/p ORIF one year ago. A recent X-ray showed marked shortening of the femoral neck with evidence of loss of bony tissue. He was sent to rehabilitation but was not able to participate with physical therapy because of the severe hip pain. He described the pain as a dull ache that is worsened with weight bearing and ambulation. The pain distribution was in the anterior and medial thigh area. He is only able to ambulate very short distances (i.e. from bed to the bathroom). His liver function tests are borderline abnormal. He has been hospitalized numerous times for infections, hepatic-renal failure, deep venous thrombosis, as well as recurrent cholangitis. His past history also includes gastroesophageal reflux, diabetes mellitus, hypothyroidism and depression. He is on numerous medications that include diuretics, antibiotics, hypertensive, opioids and immunosuppressants.

On physical exam he is jaundice with large abdomen. He is alert and orientated with fluent speech. His motor exam reveals decreased motor strength in the left leg proximally $3 / 5$ and $4 / 5$ distally. There was decrease range of motion (less than 20 degrees of hip flexion) in the left hip due to pain. The right leg is $4 / 5$ throughout. $\mathrm{He}$ has significant ascites and lower extremity edema. He was given fresh frozen plasma and had pulsed radiofrequency of the left 
obturator and femoral nerves. Immediately post procedure he had no pain and was able to move the left leg but was not able to walk due to significant deconditioning. $\mathrm{He}$ was discharged to rehabilitation unit 2 days later. In therapy he was able to participate and was eventually able to walk 200 feet with a rolling walker. He had a telephone follow-up at one month and was having significant pain relief and stated his pain ranged from 3 to 5 out of 10 verbal scale versus VAS of 9-10/10 reported prior to the procedure. He reported no side effects from the procedure. He continued to use the same amount of opioids since he had other pains. Unfortunately he passed away soon after the telephone follow-up.

\section{Case Report \#3}

A 70-year-old woman fell a month ago and since this time has complaints of right groin and thigh pain. The pain is worse with weight bearing. She has denies weakness or numbness and x-rays of the hip and low back showed degenerative changes but were otherwise unremarkable. CT scan of the pelvis revealed small superior and inferior rami fracture. She has history of insulin dependent diabetes mellitus, osteoporosis, hypothyroid, and status post laminectomy. Prior to her fall she was on oxycodone for pain but this did not significantly help her pain. On physical examination, she had decreased proximal motor strength in the right hip secondary to pain. She had pain with active and passive range of motion. She underwent radiofrequency of the obturator and femoral nerves after a successful diagnostic blockade. She had excellent relief at 4 -week follow-up $(60 \%$ reduction of pain on VAS, from 8 to 2/10). Follow-up at 2 and 3 months revealed VAS $4 / 10$ with out pain medications. She did have numbness on the lateral surface of the hip area but this did not cause any problems for her. She reports no increased activities of daily and no increased physical activity and she remains wheelchair bound. She did come of the cox-2 inhibitors that she was given initially but continued with the oxycodone which now helped with the residual pain.

\section{Case Report \#4}

A 52 year-old female with history of breast cancer with metastatic disease to the ribs and right hip developed pubic rami fracture and complained of significant hip pain. Her past history included $s / p$ right partial mastectomy, deep venous thrombosis, chemotherapy and radiation thera- py. Her pain was 7-9/10 on VAS and was in the right hip and groin area. She was on intravenous methylprednisolone, oral methadone, coumadin and anti-depressants. The patient underwent a local anesthetic block of the sensory branch of the obturator nerve supplying the right hip and had excellent relief for 2 days. She then underwent radiofrequency lesioning to these nerves. At a 1,2 and 3-month follow-up her pain in the right hip ranged from 2 to 4 out of 10 on VAS. She did not have significant improvement in function but believed that her quality of life was better since the procedure. She had no complications from this procedure. She increased dosage of the methadone but this was for the other cancer related pains.

\section{DisCUSSION}

Often hip pain can be misdiagnosed as either facet arthropathy, sacroiliac joint pain, piriformis muscle syndrome or knee pain. The reason for this is because these patients often suffer from pain in the groin, thigh, trochanter, gluteal or knee. The pain in the groin and thigh can be caused by branches of the obturator nerve or a radiculopathy of the upper lumbar nerves $\left(\mathrm{L}_{1-3}\right)$ and be the result of chronic hip flexor tendonitis, neurovascular pathology, intestinal hernias as well as internal disc disruption with somatic referral. Pain caused anterior to the hip joint area is through innervation of the femoral nerve while pain in the gluteal region is via the sciatic nerve. Gluteal pain is often mistaken for sacroiliac joint dysfunction, piriformis syndrome and or facet arthropathy. A good understanding of the mechanism, anatomical structures and a thorough history and physical exam leads to the proper diagnosis. With so many complications from total hip replacements, it seems that the procedure described in this article seems to be a reasonable choice.

In fact, in one pilot study where CT guidance was used in 15 chronic hip pain patients who were chronically ill patients with osteoarthritis and underwent a single obturator nerve block the results were remarkable. While this study reported pain relief in 7 of 15 patients for 3-11 months with only lidocaine $1 \%$. This study has not been reproduced. Furthermore, one would surmise that local anesthetics do not cause long term neurolysis and to obtain long term relief other neurolytic procedures would be necessary (4). Also, a regional anesthetic technique has been described using a blind anatomical approach to the obturator nerve and nerve to quadratus femoris, as a means of alleviating the disabling pain of chronic osteoarthritis of the hip, when arthroplasty is not available (5). However, in one study 18 patients with unilateral osteoarthrosis of the hip had a trial which involved the injection Marcaine and normal saline. Their pain, mobility and functional activity were assessed and gait characteristics analyzed, once before the injection and then at three monthly intervals. All the patients recorded a decrease in pain level within two weeks of injection, but it subsequently rose to pre-injection levels. There were no other significant general improvements after the injection. A significant increase in the maximum force at toe-off for the good leg was recorded one month after the injection. However, no further significant differences were found in the gait performance of either group of patients throughout the study. These authors concluded that the hip nerve regional blockade offers no useful relief to the pain of osteoarthrosis of the hip joint (6).

Resection of the obturator nerve in hip joint arthritis has also been performed. In a Polish study, extrapelvic obturator neuroectomy was done in 34 patients who had either a temporary or permanent contraindications for hip arthroplasty. In 21 of the 30 patients who had this procedure experienced partial or total relief of their hip pain at a 3 month follow-up (7). In a Japanese study, 14 patients with hip pain were treated by percutaneous radiofrequency lesioning of sensory branches of obturator and/or femoral nerves. All patients first underwent a diagnostic intra-articular hip joint block or articular branch block of obturator nerve with local anesthesia that was transiently effective. Then radiofrequency lesioning was performed at 75 degrees $\mathrm{C}$ to 80 degrees $C$ for 90 seconds using an RFG3B generator and Sluijter-Mehta cannulae kit (Radionics, Burlington, MA) for the obturator nerve in 9 patients and for both the obturator and femoral nerves in 5 patients. The VAS scores before and after the radiofrequency lesioning were $6.8( \pm 0.9)$ and $2.7( \pm 1.3)$, respectively. Twelve patients (86\%) reported at least 50\% relief of pain for 1 to 11 months. There were no side effects reported (1).

Although further experience and data is needed to change the current paradigm for chronic hip pain it seems reasonable to use this procedure on patients where surgery is not an option. 


\begin{tabular}{|ll|}
\hline Author Affiliation: & Mohamed Elkersh, MD \\
Atif Malik, MD & Research and Clinical Pain Fellow \\
Research and Clinical Pain Fellow & Arnold Pain Center \\
Arnold Pain Center & Department of Anesthesia \\
Department of Anesthesia & Beth Israel Deaconess Medical Center \\
Beth Israel Deaconess Medical Center & Harvard Medical School \\
Harvard Medical School & 330 Brookline Avenue \\
330 Brookline Avenue & Boston, MA-02215 \\
Boston, MA-02215 & Musa Aner, MD \\
E-mail: amalik@bidmc.harvard.edu & Instructor \\
Thomas Simopolous, MD & Arnold Pain Center \\
Instructor & Department of Anesthesia \\
Arnold Pain Center & Beth Israel Deaconess Medical Center \\
Department of Anesthesia & Harvard Medical School \\
Beth Israel Deaconess Medical Center & 330 Brookline Avenue \\
Harvard Medical School & Boston, MA-02215 \\
330 Brookline Avenue & Zahid H. Bajwa, MD \\
Boston, MA-02215 & Assistant Professor \\
& Department of Anesthesia \\
& Arnold Pain Center \\
& Beth Israel Deaconess Medical Center \\
& Harvard Medical School \\
& 330 Brookline Avenue \\
& Boston, MA-02215 \\
& \\
\hline
\end{tabular}

\section{REFERENCES}

1. Kawaguchi M, Hashizume K, Iwata $T$ et al. Percutaneous radiofrequency lesioning of sensory branches of the obturator and femoral nerves for the treatment of hip joint pain. Reg Anesth Pain Med 2001; 26:576-581.

2. Dee R. Structure and function of hip joint innervation. Ann R Coll Surg Engl 1969; 45:357-374.

3. Birnbaum K, Prescher A, Hessler $\mathrm{S}$ et al. The sensory innervation of the hip joint-an anatomical study. Surg Radiol Anat 1997; 19:371-375.

4. Heywang-Kobrunner SH, Amaya B, Okoniewski $M$ et al. CT-guided obturator nerve block for diagnosis and treatment of painful conditions of the hip. Eur Radiol 2001; 11:1047-1053.

5. James CD, Little TF. Regional hip blockade. A simplified technique for the relief of intractable osteoarthritic pain. Anaesthesia 1976; 31:1060-1067.

6. Edmonds-Seal J, Turner A, Khodadadeh S et al. Regional hip blockade in osteoarthrosis. Effects on pain perception. Anaesthesia 1982; 37:147-151.

7. Boloczko S, Bieniecki M. Resection of the obturator nerve for analgesic treatment of degenerative-deforming changes of the hip joint. Chir Narzadow Ruchu Ortop Pol 1990; 55:387-390. 